\title{
Lattice Sites of Cd in Ferroelectric $\mathrm{BaTiO}_{3}$
}

\author{
M. Dietrich, C. Camard ${ }^{\mathrm{a}}$, M. Deicher ${ }^{\mathrm{b}}$, F. Richter ${ }^{\mathrm{c}}$, V. Samokhvalov ${ }^{\mathrm{d}}$, S. Unterricker ${ }^{\mathrm{d}}$, \\ and the ISOLDE-Collaboration ${ }^{\mathrm{a}}$ \\ Technische Physik, Universität des Saarlandes, D-66041 Saarbrücken, Germany \\ ${ }^{a}$ CERN, CH-1211 Geneva 23, Switzerland \\ ${ }^{\mathrm{b}}$ Fakultät für Physik, Universität Konstanz, D-78457 Konstanz, Germany \\ ${ }^{\text {c }}$ Nicolaus-Cusanus-Gymnasium, D-51365 Bergisch-Gladbach, Germany \\ d Institut für Angewandte Physik, TU Bergakademie Freiberg, D-09596 Freiberg, Germany \\ Reprint requests to M. Di.; Fax: +41-22-767 8990, E-mail: Marc.Dietrich@cern.ch
}

Z. Naturforsch. 57 a, 613-616 (2002); received January 25, 2002

Presented at the XVIth International Symposium on Nuclear Quadrupole Interactions, Hiroshima, Japan, September 9-14, 2001.

The radioactive isotope ${ }^{111 \mathrm{~m}} \mathrm{Cd}$ was implanted into $\mathrm{BaTiO}_{3}$ in order to measure electric field gradients (EFGs) with Perturbed Angular Correlation spectroscopy (PAC). It is possible to anneal the implantation induced lattice damage to a certain extent. Then, ${ }^{111 \mathrm{~m}} \mathrm{Cd}$ probes are positioned at two sites with distinct axially symmetric EFGs characterized by the quadrupole coupling constants of 33.9(9) and 69.8(9) MHz. These EFGs can be assigned to ${ }^{111 \mathrm{~m}} \mathrm{Cd}$ at the Ti- and the Ba-sites.

Key words: Quadrupole Interaction; Perturbed Angular Correlations; Perovskites; Nuclear Probes.

\section{Introduction}

$\mathrm{BaTiO}_{3}$ has a perovskite structure and occurs at room temperature (RT) in the ferroelectric phase. The Electric field gradients (EFGs) at lattice sites reflect the non-cubic charge symmetry. They have been measured in detail for the Ba- and Ti-sites by NMR [1]. With Perturbed $\gamma \gamma$-Angular Correlation spectroscopy (PAC) the EFG can be measured with the help of radioactive probe atoms that are in most cases impurities in the material under investigation. PAC investigations on $\mathrm{BaTiO}_{3}$ have been carried out using radioactive probes that substitute for $\mathrm{Ti}:{ }^{181} \mathrm{Hf}\left({ }^{181} \mathrm{Ta}\right)$, ${ }^{111} \operatorname{In}\left({ }^{111} \mathrm{Cd}\right)[2-4]$. Recently, the influence of an external electric field on the EFG was observed [5]. These investigations shall be extended to other lattice sites than the Ti-site. In order to find a probe that substitutes $\mathrm{Ba}$, we have performed experiments after implantation of ${ }^{111 \mathrm{~m}} \mathrm{Cd}$. In this report we describe the attempts to anneal the implantation induced lattice damage. Furthermore, we discuss the measured EFGs in view of the lattice sites of ${ }^{111 \mathrm{~m}} \mathrm{Cd}$ in $\mathrm{BaTiO}_{3}$.

\section{Experimental}

$\mathrm{BaTiO}_{3}$ crystallizes in the Perovskite structure and exhibits at room temperature a tetragonal ferroelectric phase. The single crystalline sample from 'Forschungsinstitut für mineralische und metallische Werkstoffe - Edelsteine / Edelmetalle - GmbH', IdarOberstein, had a size of $2 \times 2 \mathrm{~mm}^{2}$ and a thickness of $1 \mathrm{~mm}$. The polar axis along $c$ was perpendicular to the plane. The sample had been prepared from a crystal grown with $100 \mathrm{ppm}$ Co in the melt for optoelectronics use.

In perovskites, EFGs are present at lattice sites because of the non-cubic point symmetry. The experiment gives the quadrupole coupling constant $\nu_{\mathrm{Q}}$ and the asymmetry parameter $\eta$ of the EFG. The largest component $V_{z z}$ of the EFG is directly proportional to the quadrupole coupling constant: $V_{z z}=\frac{h}{e} \frac{\nu_{\mathrm{Q}}}{Q}$ ( $h$ = Planck's constant, $e=$ elementary charge, $Q=$ quadrupole moment). $\gamma-\gamma$ PAC measurements have been performed with a standard 4-detector setup using $\mathrm{BaF}_{2}$ scintillation detectors. 

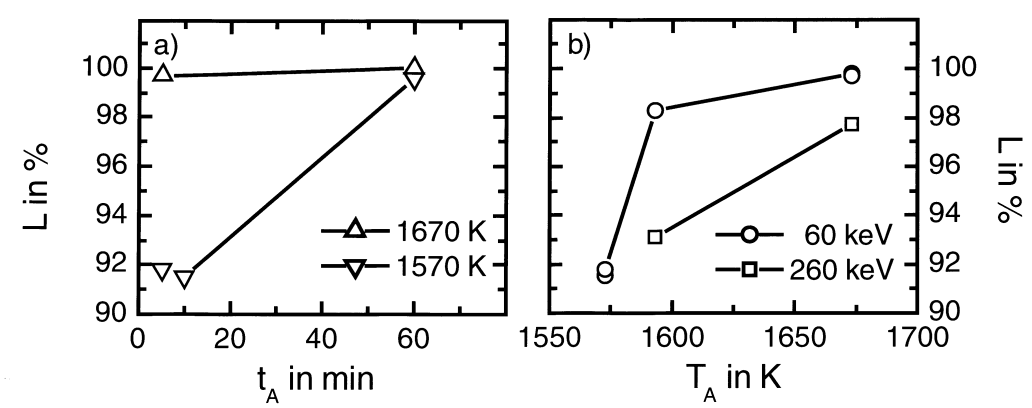

Fig. 1. Loss $L$ of activity of $\mathrm{BaTiO}_{3}$ during thermal treatment after the implantation of ${ }^{111 \mathrm{~m}} \mathrm{Cd}$. a) Loss $L$ versus annealing time $t_{\mathrm{A}}$. Parameter is the annealing temperature of $1670 \mathrm{~K}$ and $1570 \mathrm{~K}$. b) Loss $L$ versus annealing temperature $T_{\mathrm{A}}$ with the implantation energy (60 keV and $260 \mathrm{keV})$ as parameter.
We have implanted ${ }^{111 \mathrm{~m}} \mathrm{Cd}$ (half live $49 \mathrm{~min}$ ) in the single crystal at the on-line isotope separator ISOLDE at CERN with implantation energies of $60 \mathrm{keV}$ as standard beam energy and a dose of $5 \cdot 10^{12} \mathrm{~cm}^{-2}$ for each measurement. Furthermore, experiments were carried out on the high voltage platform [6] leading to a total implantation energy of $260 \mathrm{keV}$. In order to remove the implantation damage, the sample has been annealed by thermal treatment in air. It turns out that $\mathrm{Cd}$ tends to diffuse out of the material. As a prerequisite to PAC measurements, the annealing has been studied regarding the parameters temperature, duration of thermal treatment, time to cool down to room temperature (RT) and implantation energy. The dependence of the loss of activity, i. e. the fraction of radioactive probe atoms that leave the sample during annealing, on the annealing time is given in Fig. 1a) for two different temperatures. The temperature of $T=1670 \mathrm{~K}$ is known from the literature as the annealing temperature of $\mathrm{BaTiO}_{3}$ implanted with ${ }^{111}$ In [4]. Applying this procedure to $\mathrm{BaTiO}_{3}$ implanted with ${ }^{111 \mathrm{~m}} \mathrm{Cd}$ of $60 \mathrm{keV}$ leads to a loss of $100 \%$ of the activity implanted. A certain minimum temperature that leads to a partial annealing of the implantation damage is $1570 \mathrm{~K}$. The loss is similar for durations of annealing of 5 and $10 \mathrm{~min}$, but reaches $100 \%$ at a thermal treatment for 1 hour. The activity loss as a function of the annealing temperature is drawn in Fig. 1b) for implantation energies of $60 \mathrm{keV}$ and $260 \mathrm{keV}$. At these implantation energies the ions have a range of $20 \mathrm{~nm}$ and $64 \mathrm{~nm}$, respectively, as calculated by SRIM [7]. Implanting deeper into the crystal clearly decreases the loss of activity. The optimum procedure turned out to be the implantation with an energy of $260 \mathrm{keV}$ followed by the treatment at $1570 \mathrm{~K}$ for $5 \mathrm{~min}$ and subsequent slowly pulling out the sample of the furnace within five minutes. In this way $93(2) \%$ of the probes leave the sample and $7(2) \%$ are available for the measurement.
In order to compare the EFG measured with ${ }^{111 \mathrm{~m}} \mathrm{Cd}$ with those measured at host element nuclei like ${ }^{137} \mathrm{Ba}$, a Sternheimer correction is applied. For ${ }^{111 \mathrm{~m}} \mathrm{Cd}$ substituting $\mathrm{Ba}$ in $\mathrm{BaTiO}_{3}$, the largest component of the EFG at the Ba-site is calculated as $V_{z z, \mathrm{Cd}}=\frac{\left(1-\gamma_{\infty}\right)_{\mathrm{Cd}}}{\left(1-\gamma_{\infty}\right)_{\mathrm{Ba}}} V_{z z, \mathrm{Ba}}\left(V_{z z, \mathrm{X}}=\right.$ largest component of EFG observed with atom $\mathrm{X}$ at a certain lattice site, $\left(1-\gamma_{\infty}\right)_{X}=$ Sternheimer factor for an ion of element $\mathrm{X}$ [8]). The shortcomings of such considerations are well known. Nevertheless, the possible uncertainty in the quotient is much smaller than that of a single multiplier.

\section{Results and Discussion}

PAC-spectra of ${ }^{111 \mathrm{~m}} \mathrm{Cd}$ in $\mathrm{BaTiO}_{3}$ with the corresponding Fourier transforms (time independent part substracted prior to transformation) are displayed in Figure 2. The Fourier transform in Fig. 2a reveals two peaks that seem to be the fundamental and the $1^{\text {st }}$ harmonic of one interaction. This pattern changes when the orientation of the single crystalline sample is changed. The measurement of Fig. 2b was recorded with the polar $c$-axis pointing $45^{\circ}$ in between two detectors. With this orientation it is obvious that the spectrum represents two distinct EFGs at $111 \mathrm{~m} C d$-sites. The EFGs have the same orientation in the crystal lattice since the Fourier amplitudes behave similarly. The parameters of the EFG are for EFG I a quadrupole coupling constant of $\nu_{\mathrm{Q}}=33.9(9) \mathrm{MHz}$ with an asymmetry parameter of $\eta=0.0(1)$, and for EFG II $\nu_{\mathrm{Q}}=69.8(9) \mathrm{MHz}$ and $\eta=0.0(3)$. 15(2)\% of the probes are exposed to EFG I and the same fraction to EFG II. The remaining fraction of about $70 \%$ contributes to a broad frequency distribution caused by ${ }^{111 \mathrm{~m}} \mathrm{Cd}$ residing in highly distorted defect sites. These defects do not anneal during the thermal treatment that is described above. 


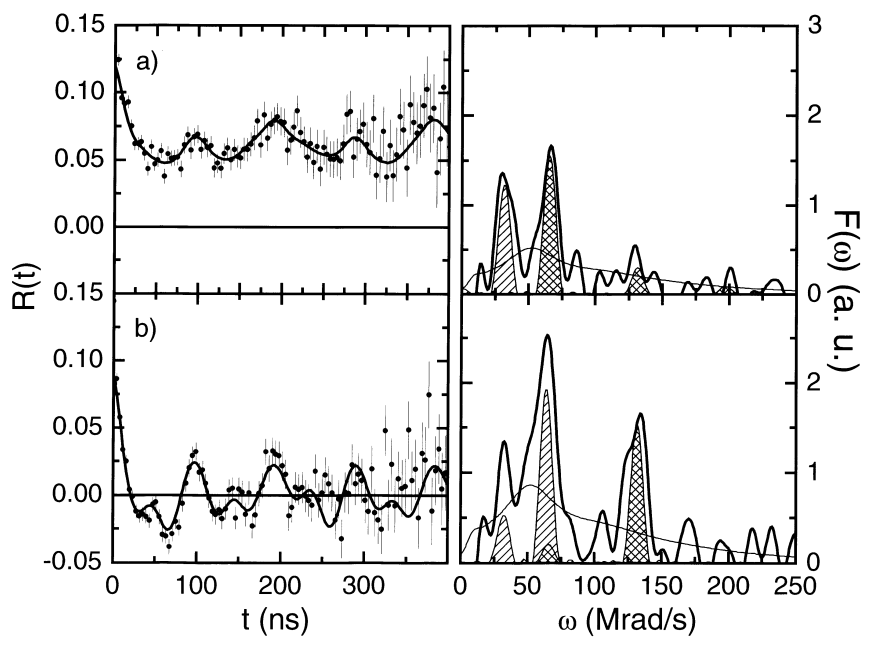

Fig. 2. PAC-spectra $R(t)$ with fit function (left) and Fourier transforms (time independent part substracted prior to transformation) $F(\omega)$ (right) for ${ }^{111 \mathrm{~m}} \mathrm{Cd}$ in $\mathrm{BaTiO}_{3}$ measured after thermal treatment. The fit takes into account two distinct EFGs and a frequency distribution. The corresponding frequencies are marked in the Fourier transform (EFG I - striped, EFG II - checkered, frequency distribution - thin line). a) Polar $c$-axis perpendicular to detector plane. b) Polar $c$-axis $45^{\circ}$ pointing in between two detectors.

Table 1. Strength of the EFGs at the Ba- and Ti-sites in $\mathrm{BaTiO}_{3}$ as measured with NMR [1]. Quadrupole coupling constants for ${ }^{111 \mathrm{~m}} \mathrm{Cd}$ calculated from the NMR values (Sternheimer antishielding) and measured with ${ }^{111 \mathrm{~m}} \mathrm{Cd}$ as well as with ${ }^{111} \mathrm{In}$ $\left({ }^{111} \mathrm{Cd}\right)[5]$.

\begin{tabular}{ccccc}
\hline $\begin{array}{c}\text { Lattice } \\
\text { site }\end{array}$ & $\begin{array}{c}\text { NMR [1] } \\
V_{z z} \text { in } 10^{20} \mathrm{~V} / \mathrm{m}^{2}\end{array}$ & $\begin{array}{c}\text { Corrected for }{ }^{111 \mathrm{~m}} \mathrm{Cd} \\
\nu_{\mathrm{Q}} \text { in } \mathrm{MHz}\end{array}$ & $\begin{array}{c}\text { Measured }{ }^{111 \mathrm{~m}} \mathrm{Cd} \\
\nu_{\mathrm{Q}} \text { in } \mathrm{MHz}\end{array}$ & $\begin{array}{c}\text { Measured }{ }^{111} \mathrm{In}\left({ }^{111} \mathrm{Cd}\right) \\
\nu_{\mathrm{Q}} \text { in } \mathrm{MHz}[5]\end{array}$ \\
\hline $\mathrm{Ba}$ & 4.35 & 2.8 & $69.8(9)$ & $33.9(9)$ \\
$\mathrm{Ti}$ & 6.82 & 42 & $34.8(1)$ \\
\hline
\end{tabular}

The quadrupole coupling constant of EFG I has exactly the same value as reported in the literature for ${ }^{111}$ In $\left({ }^{111} \mathrm{Cd}\right)$ substituting Ti $[4,5]$. This implies that half of the ${ }^{111 \mathrm{~m}} \mathrm{Cd}$ probes that occupy lattice sites with a defined EFG substitute Ti. This is surprising since Cd prefers the charge state $2+$ like $\mathrm{Ba}^{2+}$, but $\mathrm{Cd}$ has not been reported to occur in the 4+ state like the host ion $\mathrm{Ti}^{4+}$. Since the EFG II has axial symmetry and is pointing like EFG I along the polar axis of $\mathrm{BaTiO}_{3}$ we attribute EFG II to ${ }^{111 \mathrm{~m}} \mathrm{Cd}$ at the Ba-site. The EFG at the Ba-site has not been measured with PAC but was well studied with the help of ${ }^{137} \mathrm{Ba}$-NMR [1]. The EFGs of $\mathrm{Ba}$ and $\mathrm{Ti}$ in $\mathrm{BaTiO}_{3}$ are given in Table 1 , as well as the values for the quadrupole coupling constants that are expected for ${ }^{111 \mathrm{~m}} \mathrm{Cd}$. These values have been calculated using the Sternheimer correction. The quadrupole coupling constant for EFG II is much larger than the value expected for ${ }^{111 \mathrm{~m}} \mathrm{Cd}$ at the Ba-site. This fact questions the assignment of the EFG to lattice sites.

Since the assignment of the lattice sites is ambigous, one could imagine that EFG II is caused by a defect configuration. This does not seem to be the case. On the one hand the EFGs are reproducible for all spectra recorded. On the other hand, the orientation of EFG II is well defined. Defects in $\mathrm{BaTiO}_{3}$ should not have a preferred direction because after annealing the material is cooled down from the cubic phase without preferred direction to the tetragonal phase at $T=403 \mathrm{~K}$.

\section{Conclusions}

We have demonstrated the annealing of the lattice damage after implantation of ${ }^{111 \mathrm{~m}} \mathrm{Cd}$ in $\mathrm{BaTiO}_{3}$ at ISOLDE. ${ }^{111 \mathrm{~m}} \mathrm{Cd}$ is positioned at the same site as ${ }^{111} \mathrm{In}$, which is the Ti-site [4]. A second electric field gradient is assigned to the Ba-site with some uncertainty. Emission channeling experiments might clarify the situation, although this method is rather insensitive in $\mathrm{BaTiO}_{3}$ because of the high mass numbers of both $\mathrm{Ba}$ and Ti. With ab-initio calculations it will be possible in the near future to determine the relaxation around a Cd-impurity and the EFG at $\mathrm{Cd}$.

\section{Acknowledgement}

This work was financially supported by the Bundesminister für Bildung, Wissenschaft, Forschung und Technologie under Grants No. (03-DE5KO2-9) and (05 KK1TSA/7). 
[1] O. Kanert, H. Schulz, and J. Albers, Solid State Comm. 91, 465 (1994).

[2] G. L. Catchen and R. L. Rasera, Ferroelectrics (UK) 120, 33 (1991).

[3] G. Schäfer, P. Herzog, and B. Wolbeck, Z. Physik A 257, 336 (1972).

[4] M. Uhrmacher, V. V. Krishnamurty, K.-P. Lieb, A. López-García, and M. Neubauer, Z. Phys. Chem. 206, 249 (1998).
[5] M. Dietrich, J. Bartels, M. Deicher, K. Freitag, and S. Unterricker, Mat. Res. Soc. Symp. 655, CC10.11.1 (2001).

[6] H. Haas and M. Lindroos, ISOLDE Collboration, Nucl. Instrum. Methods Phys. Res. B 126, 250 (1997).

[7] J.F. Ziegler (Hrsg.), Handbook of Ion Implantation Technology, North-Holland Publishing Company, Amsterdam (1992).

[8] F. D. Feiock and W. R. Johnson, Phys. Rev. 187, 39 (1969). 